\title{
Extraction of Colour Information from Digital Images Towards Cultural Heritage Characterisation Applications
}

\author{
Woo Sik Yoo ${ }^{1}$, Jung Gon Kim ${ }^{1}$ Kitaek \\ Kang $^{1}$, and Yeongsik Yoo ${ }^{2}$ \\ ${ }^{1}$ WaferMasters, Inc., Dublin, CA, USA \\ ${ }^{2}$ Dankook University, Korea \\ Correspondence: \\ woosik.yoo@wafermasters.com
}

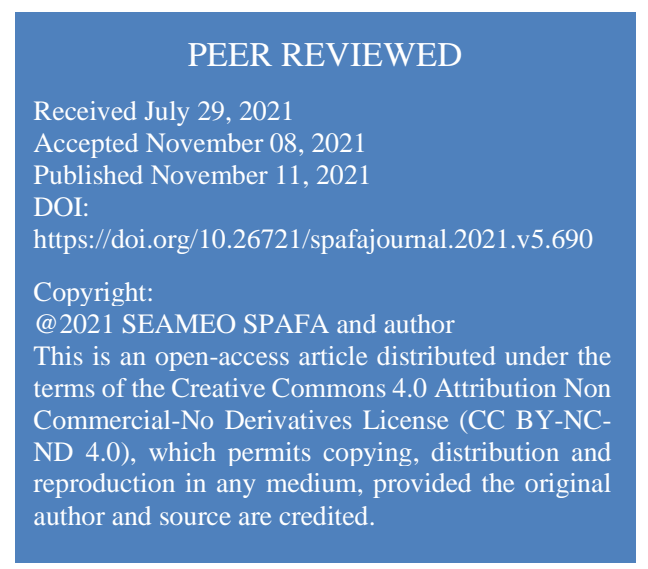

\begin{abstract}
Colour is a primary characteristic of visual perception and a very important characteristic to describe cultural heritage works in a variety of shapes, sizes and materials. To quantify colours, portable colorimeters are often carried and used for measuring colour of interest. Spatial resolution of colorimeters is manufacturer and model dependent and typically worse than $3-10 \mathrm{~mm}$ in diameter. The colour measurement has to be done on site at the time of interest. It poses very significant limitations in colorimetric characterization of cultural heritages. In this paper, the possibility of extraction of colour information from digital photographs, scanned images and video files using customized image processing/analysis software (PicMan) was investigated for cultural heritage characterization applications. As colour information extraction examples, a commercial Gouache paint colour card, a digital photograph, a portrait of a Korean scholar of Joseon Dynasty and a severely deteriorated old medical text book before and after restoration were examined. Colour information from various digital images was successfully extracted from points and regions of interest in RGB, HSV, L*a*b*, Munsell colour and hexadecimal colour code formats. The spatial resolution of colour information extraction is as small as a single pixel in a digital image.
\end{abstract}

Keywords: digital images, colour information extraction, image processing/analysis software, cultural heritage characterization

\section{Introduction}

Photographs and video images are very valuable techniques for recording conditions of cultural heritages of any kind at the time of capture. Archived photographs and video images can be very valuable resources to analyse the conditions existing at the time of capture and compare with current conditions. They can be used for comparing damage, deterioration, degradation, discoloration and effectiveness of restoration work. These become very valuable resources for not only understanding the nature of decay phenomena, but also assessing conservation conditions for improvement. Digital images and video images are very valuable resources and essential for cultural heritage applications. All archived photographs and video images can be digitized for quantitative analysis. Since we form visual perception through the stimulation of photoreceptor cells, in particular cone cells in the human eye, characterisation of colours is very important for all vision related tasks. 
The lack of user-friendly software for image-based dimensional analysis, quantitative colour analysis and statistical analysis prevents efficient utilization of born-digital or converted digital images and movies in archaeological studies. Only a very small fraction of image data is ever utilized, mainly due to the difficulties in image analysis and the labour-intensive nature of the image analysis task. To promote efficient utilization of image data it is important to develop userfriendly image processing/analysis software with necessary functions. It would be beneficial to develop user friendly, unified image processing software which can support various formats of digital images and movies (such as BMP, JPG, PNG, GIF, TIF, DM3, MOV, MP4, AVI, WMV etc.) (Yoo et al. 2019, Kim et al. 2019, Kim et al. 2021, Lee and Wi 2021, Jung et al. 2021).

In this paper, we will introduce the extraction of colour information from sample digital images using the newly developed image processing/analysis software (PicMan from WaferMasters, Inc.) and discuss possible applications in cultural heritage characterization and conservation.

\section{Types of Images Appropriate for Analysis}

A variety of image capturing devices and sensors from ultrasound (US) to X-ray wavelength range are used in the archaeological fields and labs. Besides conventional still cameras and camcorders for visible wavelength region, many different types of images and video images using different sources (different wavelength ranges) such as infrared (IR), ultraviolet (UV), X-ray, Terahertz (THz), US are used to make invisibles visible. For material characterization and geochemical instrumentation and analysis, element mapping is frequently done by energy-dispersive X-ray spectroscopy (EDS) and/or wavelength-dispersive X-ray spectroscopy (WDS).

Other than ordinary visible images, all instrumental and analytical images only contain intensity and coordinate information. They naturally become greyscale images as normally seen in X-ray and US images. For easy recognition, greyscale images are often converted to false colour images (or pseudo colour images). The range of signal intensity (or strength) is converted to pre-determined, corresponding colours to generate artificial colour (false colour or pseudo colour) images for easy recognition. Conversion of false colour images into the original greyscale images without value distortion for proper interpretation for intensity is often necessary. Keeping this requirement in mind, image processing/analysis software has been developed and customized for archaeologists, anthropologists, and conservation scientists' needs.

High resolution photographs and digitized images from old photographs, drawings, and paintings can be excellent sources for extracting colour information without spatial resolution limitation, measurement site limitation and time limitation. Colorimetric characterization from digital images can be done with very high spatial resolution (pixel by pixel) at any location and any time. Change in colours and shapes can be compared between images for planning proper protection, considering conservation options and assessing effectiveness of restoration.

\section{Image Processing/Analysis Software}

To make colour information extraction from any form of digital images and video files easier for cultural heritage characterisation applications, we have added new functions into unified image processing/analysis software, called PicMan (Yoo et al., 2019). It enables easy extraction of colour information from any or every pixel of digital images and video files in RGB, HSV, L*a*b* and Munsell colour and hexadecimal colour code formats. It can extract colour information from single 
pixels or average colour information from regions-of-interest (ROI). The extracted information can be analysed for further processing, comparisons, highlighted to communicate points, area selection for understanding, and many more operations towards cultural heritage characterization applications. It can also be used for dimension measurement from photographs and video images.

Several application examples of PicMan in conservation science have been reported. Paper foxing characterization, old Korean coin comparisons, colour analysis of ancient glass beads and colour analysis of natural solvent for reproduction of red colour from Safflower red colourant were analysed using the image processing/analysis software (PicMan) (Yoo and Kim 2017, Yoo et al. 2019, Kim et al. 2019, Kim et al. 2019, Kim et al. 2021, Lee 2021). Image comparisons for handwriting analysis were made to determine the writer of letters engraved in the corner stone of a Korean historic building during Japanese occupation (Yoo 2020). It was found to be the handwriting of Prince Ito Hirobumi (伊藤博文, 1841-1909), the first Resident-General of Japan in Korea. Artificial image modification from a very similar Yu Kil-Chun (俞吉濬, 1856-1914)’s portrait was also discovered by image analysis (Yoo and Yoo 2021). Yu Kil-Chun is a very important figure as an intellectual, writer, politician, and independence activist of Korea's late Joseon Dynasty. More example applications can be found elsewhere (Yoo et al. 2021, Jung et al. 2021).

Colour information (brightness values of RGB -red, green and blue) channel pixels at any point on the image can be read and exported in CSV format. RGB values of image files can be converted into HSV, L*a*b*, Munsell colour and hexadecimal colour code formats may be exported as comma separated value (CSV) files, as necessary. It will make quantitative colour comparisons easier and colour information exchanges between colleagues much more accurate. Schematics of colour conversion from a source image file with RGB values into different colour formats are shown in Figure 1.

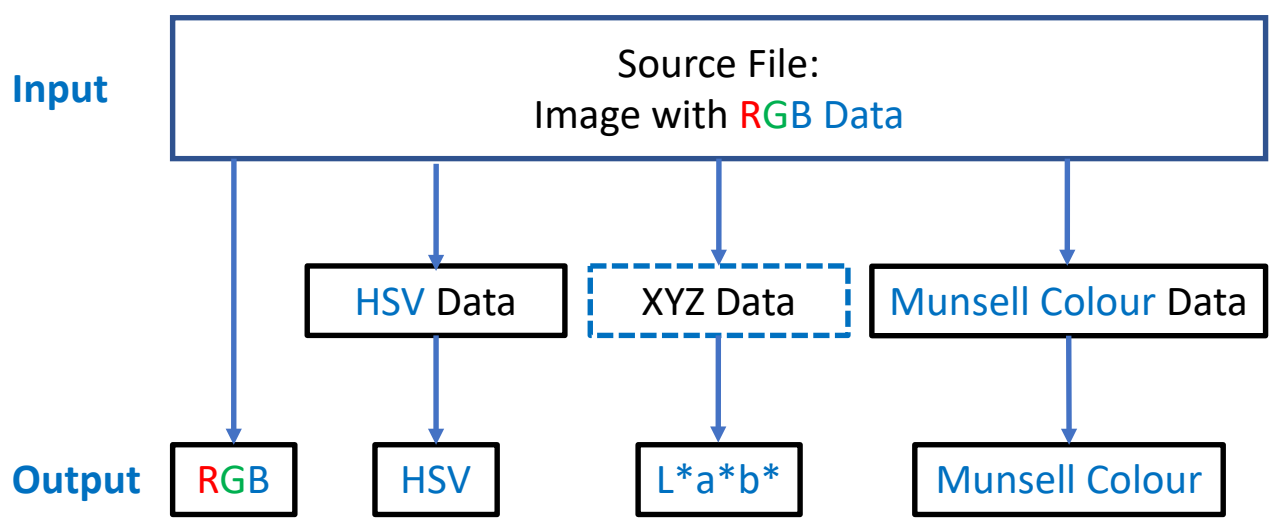

Fig. 1 Schematics of colour conversion process from a source image file with RGB format into various colour formats (coordinates). Desired colour format can be selected for individual pixels up to an entire image. Hexadecimal colour conversion is not shown. Source: Author.

\section{Colour Information Extraction Application Examples}

As colour information extraction examples using PicMan, four types of images were used in this study. A commercial Gouache paint colour card image downloaded from Amazon Singapore (Amazon Singapore 2021), a photograph from the last SEAMEO SPAFA (the Southeast Ministers of Education Organization Regional Centre for Archaeology and Fine Arts) conference in 2019, a portrait of Kang Io (姜娄五, 1788 ?) by Yi Jaegwan (李在寬, 1783-1838) from the Joseon Dynasty in Korea (National Museum of Korea 2021) and an old medical book with severe paper 
foxing and mildew, before and after treatment (Typepad 2021) were selected for colour information extraction and to demonstrate some potential applications of image processing/analysis software, PicMan.

\section{Colour Information Extraction from Colour Cards}

Figure 2 shows the commercial Gouache paint colour card image file downloaded from Amazon Singapore (Amazon Singapore 2021) opened using PicMan. Colour information at 18 points (one point per colour pad) were extracted and displayed in RGB format on screen. RGB intensity line graph (in RGB colours) with an RGB average intensity line graph (in grey colour) along the dotted red line near the left corner of the colour card can be seen from Figure 1. The intensity scale is in the range of $0-255$ for 8 -bit per channel $\left(2^{8}=256\right.$ : $\left.0-255\right)$. Near the borders between the colour pads and white background, spikes of the RGB and average RGB intensity changes can be seen. This is the effect of image compression during saving as a digital image to reduce the file size. It is more frequently observed from digital images in JPG or JPEG formats which use higher data compression ratio compared to other image file formats. It is important to save digital images into image formats with no compression or minimal compression as much as possible.

The image with colour information extraction results can be saved as a new image without overwriting on the original image file. All numerically extracted colour information can be exported as a CSV file. In the exported CSV file, measurement ID, XY coordinate information of the measurement points on the image file, colour information in RGB, HSV, L*a*b*, Munsell colour and corresponding hexadecimal colour code are exported together. To avoid screen image with crowded numbers, only one set of selected colour information is displayed. Preferred colour information display format can be selected at settings any time (before and/or after point or ROI selection). The RGB and average RGB intensity graph data can also be exported as a CSV file for duplication of graphs in preferred format and resources for further analysis. 


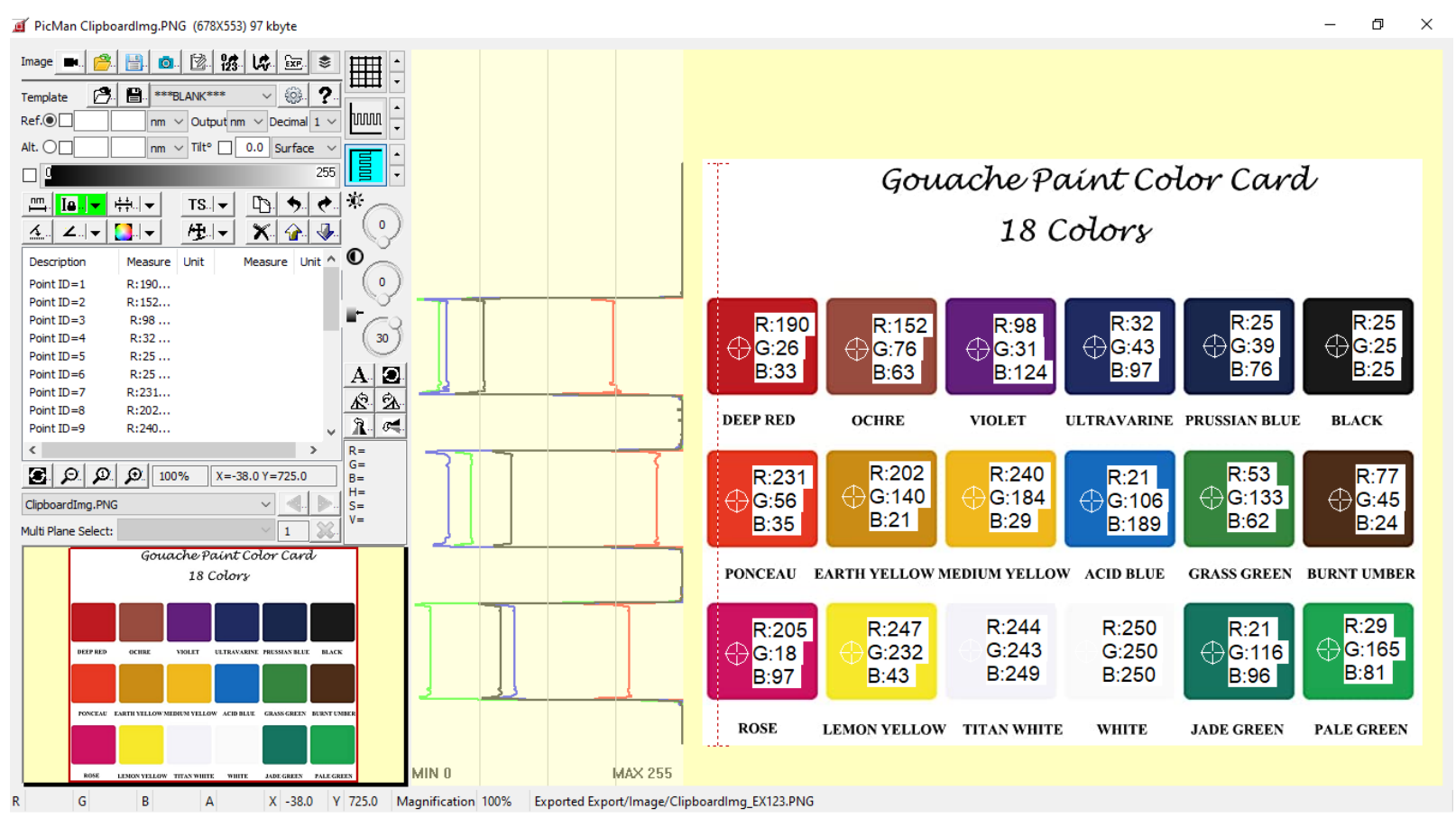

Fig. 2 A Gouache paint colour card and an example of colour information extraction of selected pixels in RGB values and individual RGB brightness (intensity) and its average intensity (brightness) line graph for a vertical line across the image (left side of colour card) using image processing/analysis software (PicMan). Source: Amazon Singapore 2021 and Author.

Figure 3 shows the colour pad portion of the Gouache paint colour card with and without extracted numerical colour information at the identical 18 points in five different formats. Figure 3 (a) shows the original image without displaying numerical colour information. Figures 3 (b) - (f) show hexadecimal colour codes, RGB values, HSV values, $\mathrm{L}^{*} \mathrm{a} \mathrm{b}^{*}$ values and Munsell colour format, respectively. Correlation between different colour formats for selected colours can be found by looking at the summarized image. To make the comparison easier, colour information for all 18 points were exported as CSV file as shown in Table 1. Measurement point ID and their XY coordinate information are exported together to locate the exact location of measurement points (or pixels) on the image. Colour information of all pixels on the image can be extracted and exported for detailed analysis, if necessary. Various types of graphs, including histograms using extracted colour information on RGB, HSV, L*a*b* and Munsell colour formats on images, can be generated for further analysis. 


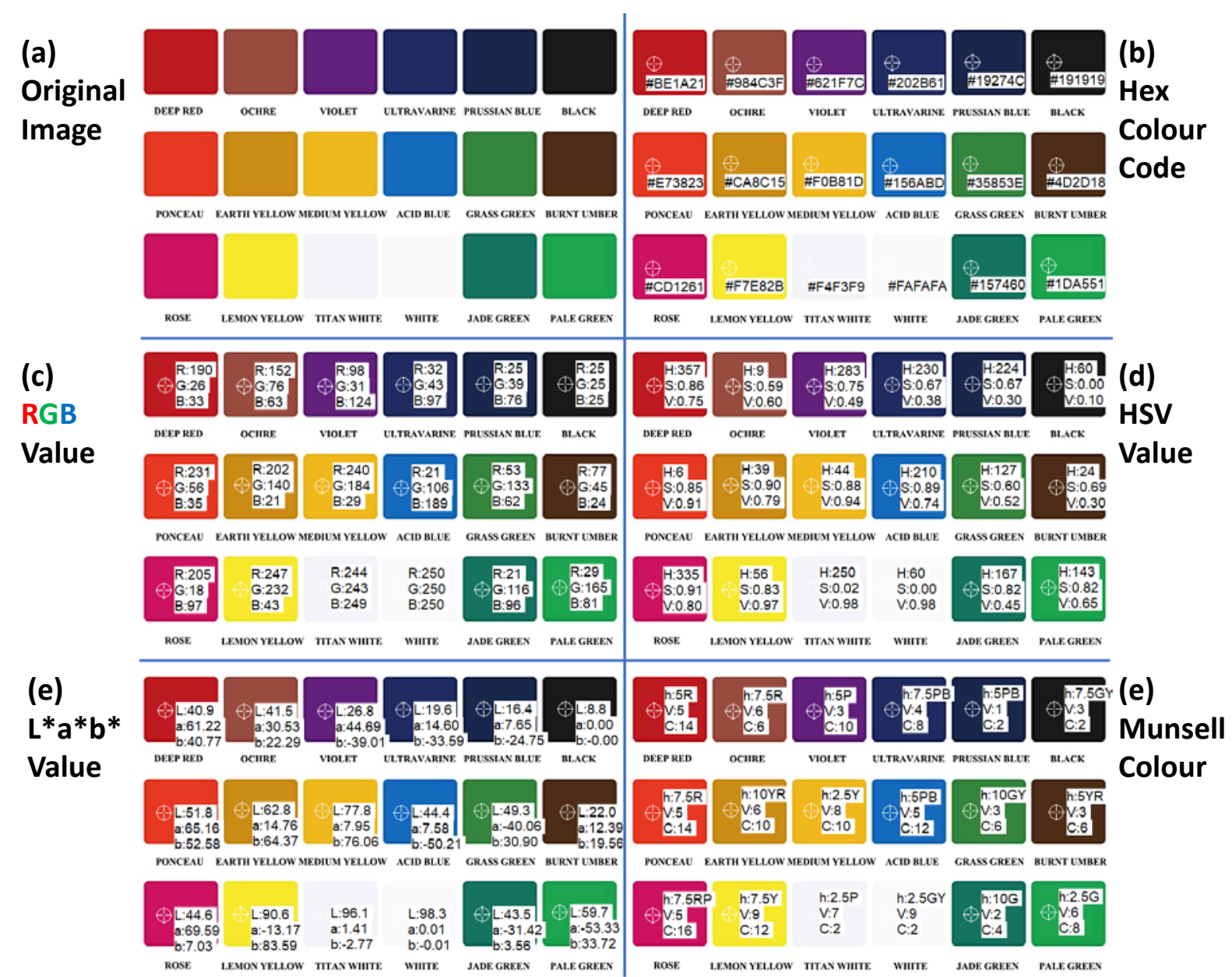

Fig. 3 (a) A Gouache paint colour card and examples of colour information extraction from 18 different solid boxes with different colours in (a) hexadecimal colour code, (c) RGB values, (d) HSV values, (e) L*a*b* values and (f) Munsell colours using PicMan. Source: Amazon Singapore 2021 and Author.

\section{Colour Calibration of Photographs}

Colour calibration charts from other suppliers such as X-Rite ColorCheckers, Kodak colour calibration charts, and Tiffen colour separation guides with grey scales were also tested to compare colour information extracted from them. For comparing $\mathrm{L}^{*} \mathrm{a} \mathrm{b}^{*}$ values extracted from photographs of colour calibration charts using PicMan with actual measurement data on colour calibration charts, $\mathrm{L}^{*} \mathrm{a} * \mathrm{~b} *$ values on all colour pads on colour calibration charts were measured using a Konica Minolta CR-300 Chroma meter and an x-rite i1Photo Pro 2 colour measurement device. The minimum sampling area for colour measurements for the Konica Minolta CR-300 Chroma meter and x-rite i1Photo Pro 2 device are $19.6 \mathrm{~mm}^{2}$ and $9.6 \mathrm{~mm}^{2}$ (5 mm and $3.5 \mathrm{~mm}$ diameter), respectively. The typical spatial resolution of these devices is in the range of $3-10 \mathrm{~mm}$ in diameter. It is impossible to measure colour of objects smaller than the measurement area. To make meaningful colour measurements, the objects must have their size greater than the sampling area (19.6 $\mathrm{mm}^{2}$ (or $5 \mathrm{~mm}$ diameter) for the Konica Minolta CR-300 Chroma meter (i. e. colourimeter) and $9.6 \mathrm{~mm}^{2}$ (or $3 \mathrm{~mm}$ diameter) for the $\mathrm{x}$-rite i1Photo Pro 2 device) uniform colour across the entire sampling area. Any objects either smaller than the sampling area or with non-uniform colours within the sampling area are not suitable for colour measurement using the conventional colour measurement devices. Thus, the colour of very small areas or fine lines such as textures on clothes, 
hair, whiskers and decorative hatch patterns in paintings were not properly characterised, even with the chromameter and colour measurement devices.

\begin{tabular}{|c|c|c|c|c|c|c|c|c|c|c|c|c|c|c|}
\hline \multirow[b]{2}{*}{ I.D. } & \multicolumn{2}{|c|}{ Coordinate } & \multicolumn{3}{|c|}{ RGB Value } & \multicolumn{3}{|c|}{ Colours in HSV } & \multicolumn{3}{|c|}{ Colours in $\mathrm{L}^{*} \mathrm{a}^{*} \mathrm{~b}^{*}$} & \multicolumn{3}{|c|}{ Munsell Colour } \\
\hline & $\mathrm{X}$ & $\mathrm{Y}$ & $\mathrm{R}$ & G & B & $\mathrm{H}$ & $\mathrm{S}$ & $\mathrm{V}$ & $\mathrm{L}$ & a & $\mathrm{b}$ & $\mathrm{h}$ & V & C \\
\hline 1 & 34 & 178 & 190 & 26 & 33 & 357.4 & 0.86 & 0.75 & 40.90 & 61.22 & 40.77 & $5 R$ & 5 & 14 \\
\hline 2 & 145 & 179 & 152 & 76 & 63 & 8.8 & 0.59 & 0.60 & 41.50 & 30.53 & 22.29 & $7.5 R$ & 6 & 6 \\
\hline 3 & 259 & 179 & 98 & 31 & 124 & 283.2 & 0.75 & 0.49 & 26.80 & 44.69 & -39.01 & $5 P$ & 3 & 10 \\
\hline 4 & 369 & 177 & 32 & 43 & 97 & 229.8 & 0.67 & 0.38 & 19.60 & 14.60 & -33.59 & 7.5PB & 4 & 8 \\
\hline 5 & 482 & 176 & 25 & 39 & 76 & 223.5 & 0.67 & 0.30 & 16.40 & 7.65 & -24.75 & 5PB & 1 & 2 \\
\hline 6 & 597 & 176 & 25 & 25 & 25 & 60.0 & 0.00 & 0.10 & 8.80 & 0.00 & 0.00 & $7.5 \mathrm{GY}$ & 3 & 2 \\
\hline 7 & 32 & 322 & 231 & 56 & 35 & 6.4 & 0.85 & 0.91 & 51.80 & 65.16 & 52.58 & $7.5 \mathrm{R}$ & 5 & 14 \\
\hline 8 & 142 & 318 & 202 & 140 & 21 & 39.4 & 0.90 & 0.79 & 62.80 & 14.76 & 64.37 & 10YR & 6 & 10 \\
\hline 9 & 255 & 319 & 240 & 184 & 29 & 44.1 & 0.88 & 0.94 & 77.80 & 7.95 & 76.06 & $2.5 \mathrm{Y}$ & 8 & 10 \\
\hline 10 & 366 & 322 & 21 & 106 & 189 & 209.6 & 0.89 & 0.74 & 44.40 & 7.58 & -50.21 & $5 P B$ & 5 & 12 \\
\hline 11 & 479 & 319 & 53 & 133 & 62 & 126.7 & 0.60 & 0.52 & 49.30 & -40.06 & 30.90 & $10 G Y$ & 3 & 6 \\
\hline 12 & 600 & 321 & 77 & 45 & 24 & 23.8 & 0.69 & 0.30 & 22.00 & 12.39 & 19.56 & $5 Y R$ & 3 & 6 \\
\hline 13 & 32 & 466 & 205 & 18 & 97 & 334.7 & 0.91 & 0.80 & 44.60 & 69.59 & 7.03 & 7.5RP & 5 & 16 \\
\hline 14 & 140 & 465 & 247 & 232 & 43 & 55.6 & 0.83 & 0.97 & 90.60 & -13.17 & 83.59 & $7.5 Y$ & 9 & 12 \\
\hline 15 & 252 & 463 & 244 & 243 & 249 & 250.0 & 0.02 & 0.98 & 96.10 & 1.41 & -2.77 & $2.5 \mathrm{P}$ & 7 & 2 \\
\hline 16 & 361 & 464 & 250 & 250 & 250 & 60.0 & 0.00 & 0.98 & 98.30 & 0.01 & -0.01 & $2.5 \mathrm{GY}$ & 9 & 2 \\
\hline 17 & 480 & 465 & 21 & 116 & 96 & 167.4 & 0.82 & 0.45 & 43.50 & -31.42 & 3.56 & 10G & 2 & 4 \\
\hline 18 & 589 & 462 & 29 & 165 & 81 & 142.9 & 0.82 & 0.65 & 59.70 & -53.33 & 33.72 & $2.5 \mathrm{G}$ & 6 & 8 \\
\hline
\end{tabular}

Table 1 Summary of extracted colour information from 18 boxes in the Gouache paint colour card. (Measurement I.D. and coordinate information of extraction points on the image are available with colour information in various formats.) Source: Author.

\section{Definition of Colour Difference}

The RGB format was intended for convenient use with electronic systems for image processing and display devices. Unfortunately, it does not align with how we actually perceive colour. By simply comparing the Euclidean distance difference between the red, green and blue intensities of RGB values of a pixel, colour difference cannot be determined. To compare colours, RGB to L*a*b* conversion is required. The colour difference between two colours is defined as a single number Delta-E ( $\Delta \mathrm{E}$ or $\Delta E_{a b}^{*}$ ) in the $\mathrm{L}^{*} \mathrm{a}^{*} \mathrm{~b} *$ colour space. The $\Delta E_{a b}^{*}$ can be calculated using the following formula.

$$
\Delta E_{a b}^{*}=\sqrt{\left(L_{2}^{*}-L_{1}^{*}\right)^{2}+\left(a_{2}^{*}-a_{1}^{*}\right)^{2}+\left(b_{2}^{*}-b_{1}^{*}\right)^{2}}
$$

Mathematically, a $\Delta \mathrm{E}$ of 1.0 is the smallest colour difference the human eye can notice. Thus, any $\Delta \mathrm{E}$ less than 1.0 is imperceptible by human eyes. However, $\Delta \mathrm{E}$ greater than 1 are perfectly acceptable, maybe even unnoticeable in some colours, particularly dark colours. The same $\Delta \mathrm{E}$ colour difference between two yellows and two blues may not appear to be the same difference to human eyes, but it is the best known and practical quantification method for the colour differences to date. On a typical scale, the DE Value will range from 0 to $100 . \Delta E_{a b}^{*} \approx 2.3$ corresponding to a JND (just noticeable difference) (Sharma 2003). The distance between two colours is known as the $\Delta \mathrm{E}$, and has been accepted as an industry standard that is overseen by the International Commission on Illumination. From a practical perspective, the average human eye cannot detect any colour differences with a $\Delta \mathrm{E}<3$, and an exceptionally trained and sensitive human eye will only be able to 
perceive colour differences with a $\Delta \mathrm{E} \geq 1$ (Nix Color Sensor 2021). General guidelines for the relationship between $\Delta \mathrm{E}$ value and perception by human eyes are summarized in Table 2 .

\begin{tabular}{ll}
\hline DE Value & Perception by human eyes \\
\hline $1<\mathrm{DE}$ & Not perceptible by human eyes. \\
$1<\mathrm{DE}<2$ & Perceptible through close observation. \\
$2<\mathrm{DE}<10$ & Perceptible at a glance. \\
$11<\mathrm{DE}<49$ & Colours are more similar than opposite. \\
$\mathrm{DE}=100$ & Colours are exact opposite. \\
\hline
\end{tabular}

Table 2 Human perception versus colour difference DE values. Source: Schuessler 2021 and Author.

The colour difference $\Delta \mathrm{E}$ among chroma meter, colour measurement device and PicMan on the same colour patches were found to be in the range of $5.0-9.5$. The colour difference between colour patches were very similar $(\Delta \mathrm{E} \leq 2)$ among all three methods, suggesting possible improvement after cross calibration of absolute $\mathrm{L}^{*} \mathrm{a} \mathrm{b}^{*}$ values. The feasibility of PicMan for colour information extraction from photographs has been successfully demonstrated.

\section{Colour Information Extraction from Normal Photographs}

Figure 4 shows a colour information extraction example from a digital photograph. The photograph was taken at the reception hall at the previous conference in 2019 (SEAMEO SPAFA Conference 2019) by one of the authors. Extraction of colour information of selected points of interest in hexadecimal colour format is demonstrated. Colour information in different formats can be selected as needed. Extracted colour information in several formats can be exported together with measurement IDs and XY coordinate information, at any time, as seen in Table 1. To measure colour information of any objects traditionally, the chroma meter or colour measurement device must contact the objects. The objects cannot be moving at all. In other words, colour information cannot be extracted from moving objects. The merit of this technique is the non-contact, nondestructive and off-line colour measurement capability of any objects moving or not.

However, there is a great concern about the truth of colours in ordinary photographs taken from uncontrolled lighting or illumination conditions. Today's most commercially available digital cameras have built-in automatic brightness, contrast, white balance, aperture, and exposure time control functions to adapt to various lighting conditions (from dark to bright and indoor to outdoor). Most of the time, we are able to capture photographs with very vivid and realistic colours. If we do not see the significant difference in colours of actual objects and photographic images, it is safe to assume the colour difference $\Delta \mathrm{E}$ should be $<10$ as seen from the table 2 . If brightness of photographs is altered, it will also affect $\Delta \mathrm{E}$ values. The brightness of a photograph affects the $\mathrm{L}^{*}$ component and results in increase of $\Delta \mathrm{E}$ values even with constant $\mathrm{a}^{*}$ and $\mathrm{b}^{*}$ values. Thus, the proper control of illumination and colour correction is required for extracting accurate colour information. It is a matter of choice whether we want to collect very precise colour information or capability of freedom in colour information collection from very casual photographs and video images. It is always better to have more than one option as the traditional colour measurement practice. PicMan also has colour correction functionality based on white balance and contrast in RGB intensity histogram and distribution. Contrast stretching technique can also be applied for colour correction (Yoo and Yoo 2021). 


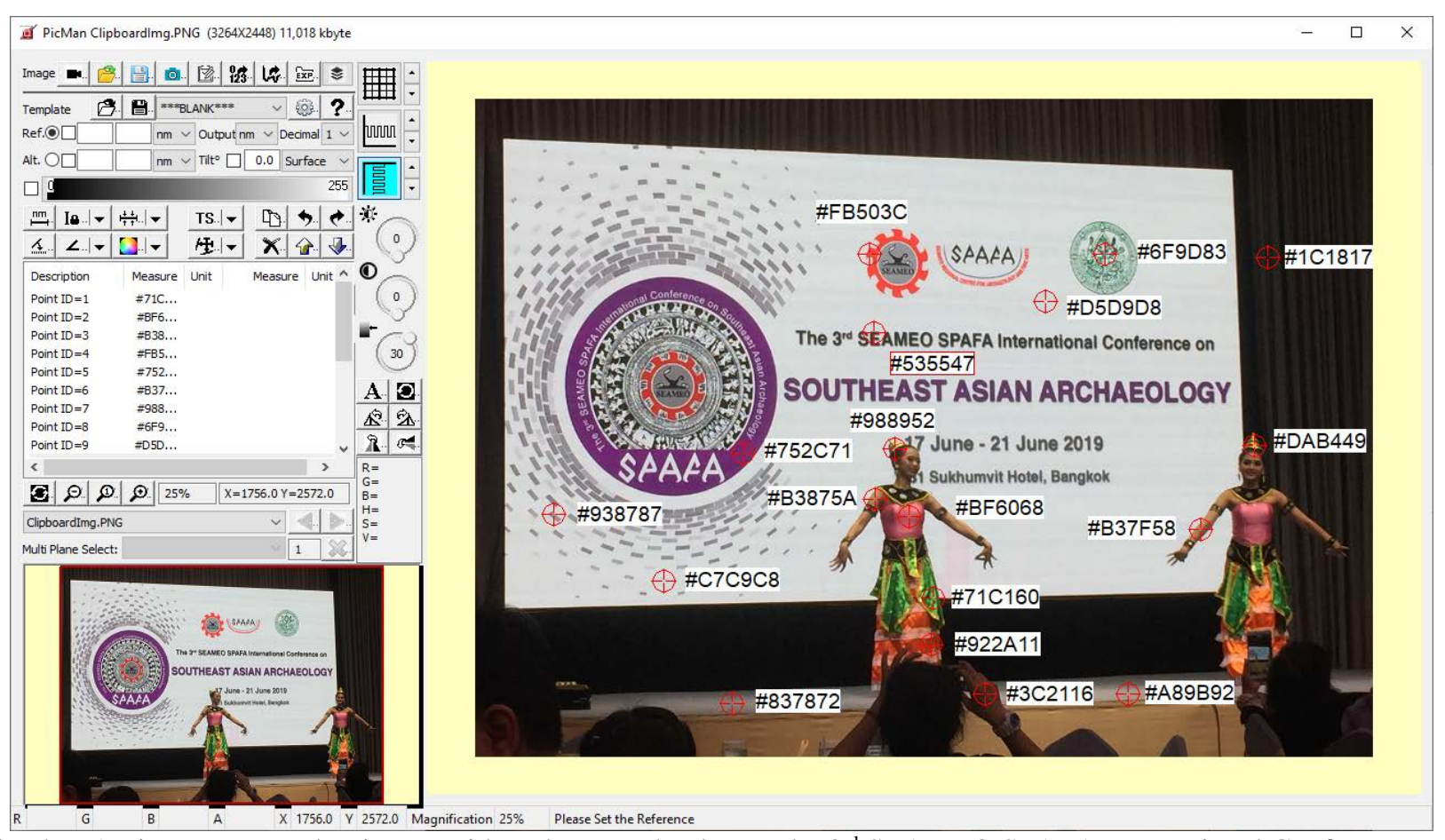

Fig. 4 A PicMan screenshot image with a photograph taken at the $3^{\text {rd }}$ SEAMEO SPAFA International Conference (Bangkok, June 2019) and hexadecimal colour codes at selected position on the image. Source: Author.

\section{Colour Information Extraction from Colour Corrected Photographs}

A Portrait of Kang Io (姜彜五, 1788 ?) in Joseon Dynasty by Yi Jaegwan (李在寬, 1783-1837) is shown in Figure 5 (National Museum of Korea 2021 and Author). The portrait was photographed with a standard colour chart and colour corrected by post image processing. The colour chart portion of the image was intentionally cropped for space limitations in this paper. Colour information at twenty (20) points on the portrait were measured in $\mathrm{L}^{*} \mathrm{a} * \mathrm{~b} *$ format. For easy comparisons, the original image without colour information display and the image with extracted colour information are shown side by side. If there are strange-coloured spots in very small size, it would not be possible to determine the colour information using colorimeters because they require at least 3-10 mm measurement area depending on specific manufacturers and models due to the nature of measurement principle. It has very poor spatial resolution. Colour information on small spots surrounded by different colours cannot be measured by colorimeters. Colorimeters are only useful if the measurement area of interest is uniform in colour and the size is bigger than the spatial resolution (or probing diameter). In the case of PicMan, colour information on every pixel can be extracted. Colour of a very thin hair can be measured without any problem. This is because image analysis is done pixel by pixel. Image analysis techniques can add great convenience as well as higher spatial resolution by principle. If we take photographs with standard colour information, it can be preciously calibrated. It is time to utilize available technology and apply it in daily practice. 


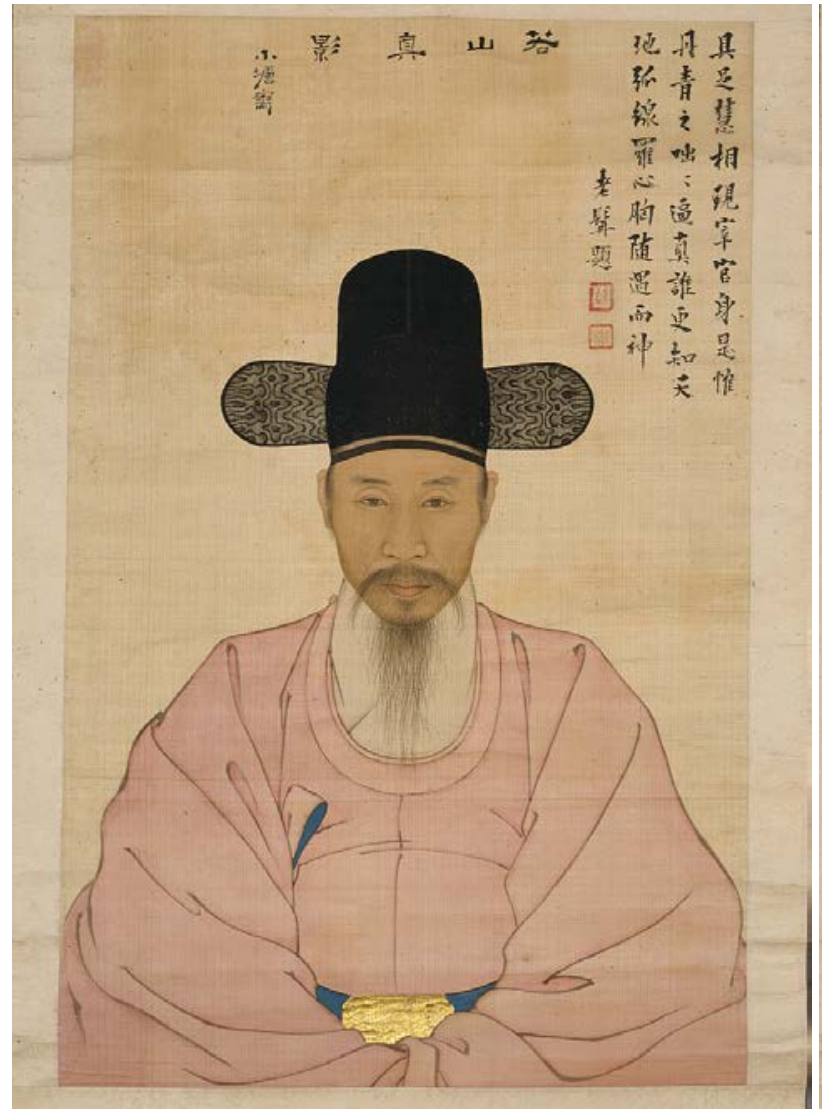

(a)

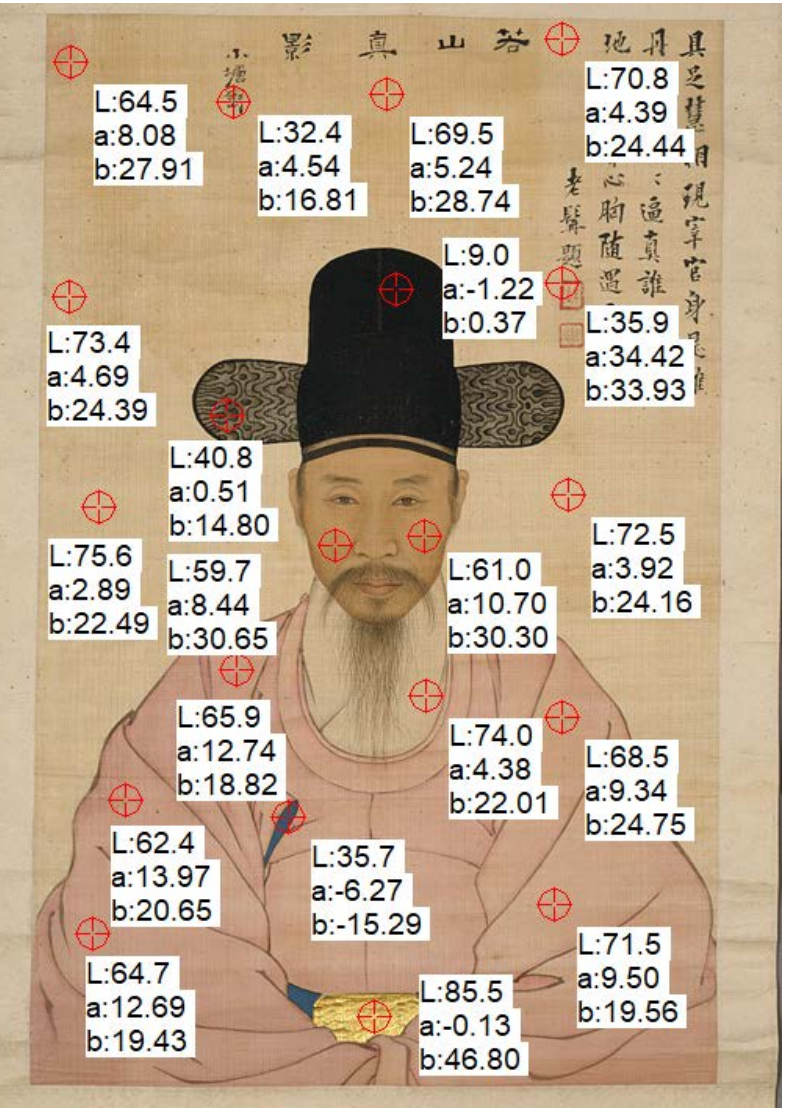

(b)

Fig. 5 (a) A Portrait of Kang Io (姜彜五, 1788 ?) by Yi Jaegwan (李在寬, 1783-1837) and (b) colour information (in $\mathrm{L}^{*} \mathrm{a} \mathrm{b}^{*}$ format) extracted from selected points using PicMan. Source: National Museum of Korea 2021 and Author.

Figure 6 shows a page from an old medical book before and after paper foxing and mildew removal. The original image showed severe paper foxing and dark spots in various sizes and colours. Colour of the page has been changed dramatically after paper foxing and mildew removal treatment. To compare the effect of treatment in terms of colour change, eight points per image were measured and colour information extracted in $\mathrm{L}^{*} \mathrm{a} * \mathrm{~b} *$ format. The same locations were selected as much as possible for fair comparisons. L* values, representing lightness (or brightness) have been increased significantly after the treatment as seen in Figure 6.

Colour information from eight (8) points per image were extracted and exported as a CSV file. Total number of measurements was 16 (8 per image). Measurement IDs L1-L8 are for the original image (left side image) and measurement IDs R1-R8 are for the image after treatment (right side image). The extracted colour information was summarized in Table 2. 


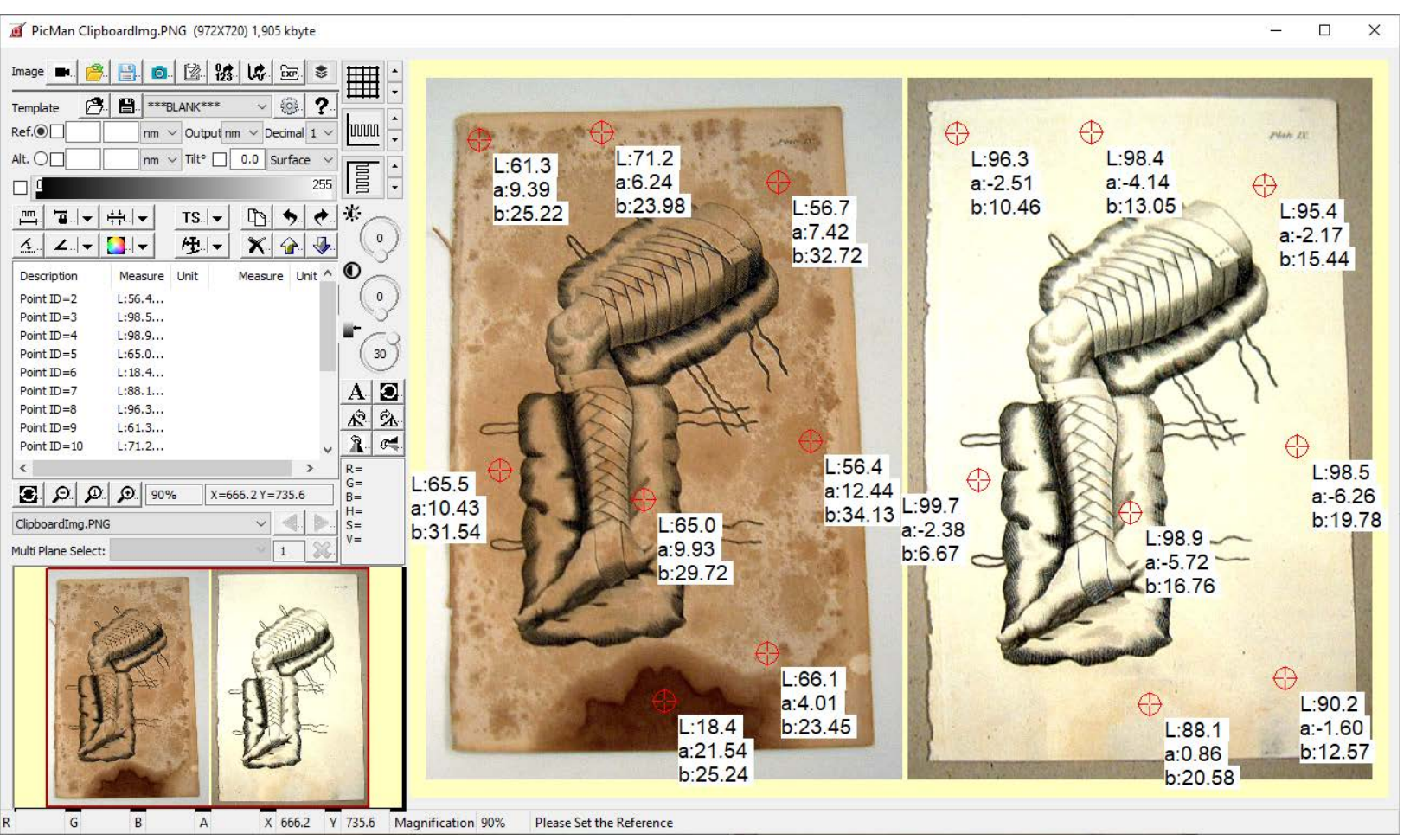

Fig. 6 Colour information (in L*a*b* format) extracted from selected points on a page (with heavy paper foxing and mildew) of a medical book before and after treatment. Source: Typepad 2021 and Author.

Bar graphs on colour information (in RGB format) extracted from 16 points from two images, before and after foxing and mildew removal, were shown in Figure 7 (a) and (b). Eight (8) points (L1-L8) in Figure 7 (a) are from the original image and eight (8) points (R1-R8) in Figure 7 (b) are from the image after treatment). After the treatment, intensity (brightness) of all channels (RGB channels) were significantly increased. Similar graphs can be plotted in HSV, L*a*b* and Munsell colour values for easy comparison in objective ways using quantitatively extracted colour information on the points of interest.

All colour information extraction examples shown here are from single pixel data for demonstrating higher spatial resolution due to the nature of image analysis technique. ROI can be set to any size and shape, from single pixel to an entire image, depending on the purpose of the analysis. It can provide very flexible solutions in many aspects, including surprising discovery from old photos and video files.

By comparing images from different times, chronological changes in colour can be quantified for understanding deterioration, degradation, and paper foxing mechanisms. The graphs showing changes in $\mathrm{L}^{*} \mathrm{a} \mathrm{b}^{*}$ values and $\mathrm{DE}$ as a function of time at the same location or average of selected area on the photographs can provide great insight into colour change and its rate over time and effectiveness of conservation environment. Two images in Figure 6 can be a great quantification example for colour change and effectiveness of colour restoration treatment. Based on the understanding, an effective conservation and restoration strategy can be formulated. After implementation, periodic inspection and evaluation can provide insight into the effectiveness of the action employed. 


\begin{tabular}{|c|c|c|c|c|c|c|c|c|c|c|c|c|c|c|}
\hline \multirow[b]{2}{*}{ I.D. } & \multicolumn{2}{|c|}{ Coordinate } & \multicolumn{3}{|c|}{ RGB Value } & \multicolumn{3}{|c|}{ Colours in HSV } & \multicolumn{3}{|c|}{ Colours in $L^{*} a^{*} b *$} & \multicolumn{3}{|c|}{ Munsell Colour } \\
\hline & $\mathrm{X}$ & $\mathrm{Y}$ & $\mathrm{R}$ & G & B & $\mathrm{H}$ & $\mathrm{S}$ & $\mathrm{V}$ & $\mathrm{L}$ & a & $\mathrm{b}$ & $\mathrm{h}$ & $\mathrm{V}$ & C \\
\hline L1 & 395 & 372 & 173 & 126 & 76 & 30.9 & 0.56 & 0.68 & 56.40 & 12.44 & 34.13 & 7.5YR & 7 & 8 \\
\hline L2 & 224 & 432 & 192 & 150 & 105 & 31.0 & 0.45 & 0.75 & 65.00 & 9.93 & 29.72 & $10 Y R$ & 5 & 2 \\
\hline L3 & 245 & 639 & 79 & 30 & 5 & 20.3 & 0.94 & 0.31 & 18.40 & 21.54 & 25.24 & $7.5 \mathrm{YR}$ & 1 & 2 \\
\hline L4 & 54.5 & 63.3 & 179 & 141 & 104 & 29.6 & 0.42 & 0.70 & 61.30 & 9.39 & 25.22 & 7.5YR & 6 & 6 \\
\hline L5 & 180 & 55.6 & 201 & 169 & 131 & 32.6 & 0.35 & 0.79 & 71.20 & 6.24 & 23.98 & 10YR & 5 & 2 \\
\hline L6 & 350 & 590 & 183 & 157 & 119 & 35.6 & 0.35 & 0.72 & 66.10 & 4.01 & 23.45 & $10 Y R$ & 7 & 4 \\
\hline L7 & 75.6 & 402 & 195 & 151 & 103 & 31.3 & 0.47 & 0.76 & 65.50 & 10.43 & 31.54 & 7.5YR & 7 & 6 \\
\hline L8 & 361 & 107 & 166 & 130 & 79 & 35.2 & 0.52 & 0.65 & 56.70 & 7.42 & 32.72 & 10YR & 6 & 6 \\
\hline R1 & 894 & 378 & 254 & 253 & 212 & 58.6 & 0.17 & 1.00 & 98.50 & -6.26 & 19.78 & $10 Y$ & 9 & 2 \\
\hline $\mathrm{R} 2$ & 723 & 446 & 254 & 254 & 219 & 60.0 & 0.14 & 1.00 & 98.90 & -5.72 & 16.76 & $2.5 \mathrm{GY}$ & 9 & 2 \\
\hline R3 & 743 & 642 & 238 & 219 & 182 & 39.6 & 0.24 & 0.93 & 88.10 & 0.86 & 20.58 & $2.5 \mathrm{Y}$ & 7 & 2 \\
\hline R4 & 545 & 56.7 & 248 & 245 & 224 & 52.5 & 0.10 & 0.97 & 96.30 & -2.51 & 10.46 & $7.5 \mathrm{Y}$ & 8 & 2 \\
\hline R5 & 683 & 55.6 & 253 & 252 & 225 & 57.9 & 0.11 & 0.99 & 98.40 & -4.14 & 13.05 & $7.5 \mathrm{Y}$ & 9 & 2 \\
\hline R6 & 882 & 616 & 234 & 227 & 203 & 46.5 & 0.13 & 0.92 & 90.20 & -1.60 & 12.57 & $5 Y$ & 8 & 4 \\
\hline R7 & 567 & 412 & 255 & 255 & 241 & 60.0 & 0.05 & 1.00 & 99.70 & -2.38 & 6.67 & $2.5 \mathrm{GY}$ & 9 & 2 \\
\hline R8 & 861 & 110 & 250 & 242 & 212 & 47.4 & 0.15 & 0.98 & 95.40 & -2.17 & 15.44 & $5 Y$ & 9 & 2 \\
\hline
\end{tabular}

Table 2 Summary of extracted colour information from 16 points from two images, before and after foxing and mildew removal, (8 points (L1-L8) from the original image and 8 points (R1-R8) from the image after treatment) Source: Author.

(a)

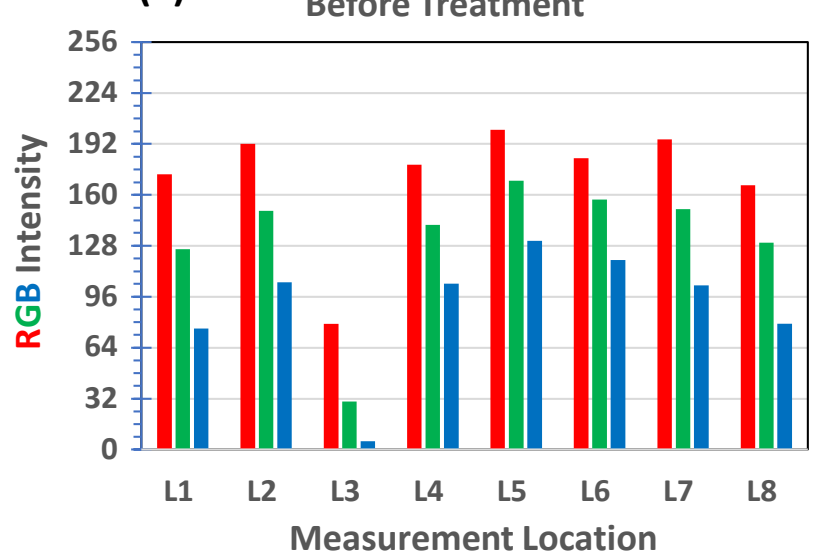

(b)

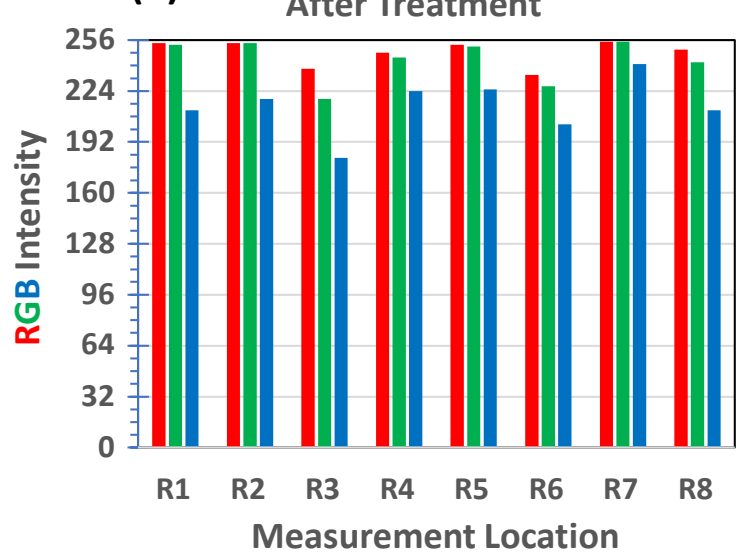

Fig. 7 Bar graphs on colour information (in RGB format) extracted from 16 points from two images (a) before and (b) after foxing and mildew removal (8 points (L1-L8) from the original image and 8 points (R1-R8) from the image after treatment) Source: Author.

\section{Perspectives}

Image analysis software, PicMan, was customized for cultural heritage characterization applications and specifically for quantitative colour information extraction from digital images and video files. It can be used for archaeological and conservation science applications. Unlike conventional colour measurement techniques using chromameters or colour measurement devices with spatial resolution of $3-10 \mathrm{~mm}$ in diameter, the spatial resolution of colour information extraction as small as a single pixel in a digital image can be achieved. The colour information extraction from regular digital photographs and video images, with or without colour correction, can be used as a valuable 
quantifiable colour information source. The conventional colorimetric measurement requires the colorimeter implementation at the time of colour measurement. It also requires contact to the objects of interest. The objects must be stationary and cannot be dynamic.

In case of digital image analysis, colour information extraction can be done from any images at anywhere and anytime. It is a non-contact colour measurement technique and can be used for dynamic (moving) objects as well. The colour information can be extracted from historic photographs or photographs taken from excavation sites or surveys years ago. The ability to extract and export numerical colour information from various digital images, from single pixels to the entire image in RGB, HSV, L*a*b*, Munsell colour and hexadecimal colour code formats are extremely valuable. They give greater flexibility and traceability in colour information acquisition and management, from the level and accuracy of single pixel to the entire image. The elimination of sample size limitations due to the spatial resolution and sampling diameter of traditional colour measurement techniques provide an additional benefit. This can greatly help us understand colour information of cultural heritage and form effective strategies for preservation, conservation and restoration activities. Further colour calibration study to improve colour matching with the conventional technique will be conducted.

\section{Acknowledgment}

The authors would like to express their special thanks to Prof. Young Hoon Jo of Dept. of Cultural Heritage Conservation Science, Kongju National University, Choongnam, Korea for his cooperation and in-depth discussions on colour measurements and practices in the field of cultural heritage conservation science.

\section{Bibliography or References}

Amazon Singapore (2021) Gouache-Vibrant-Portable-Painting-Watercolor. Available at: https://www.amazon.sg/Gouache-Vibrant-Portable-Painting-Watercolor/dp/B07XCL1RXP [accessed 9 July 2021].

Jung, YH, Kim, G and Yoo, WS (2021) Study on Distortion Compensation of Underwater Archaeological Images Acquired through a Fisheye Lens and Practical Suggestions for Underwater Photography - A Case of Taean Mado Shipwreck No. 1 and No. 2. Journal of Conservation Science, 37(4): 312-321.

Kim, EA, Kim, DS, Hyen, JH and Kim, GH (2019) Study on Material Characteristic Evaluation of Sangpyeongtongbo Coins in Joseon Dynasty Using Non-Destructive Analysis. Science and Engineering of Cultural Heritage, 14(1): 23-30.

Kim, EA, Lee, JH and Kim, GH (2021) A Characteristic Analysis of Glass Beads in Geumgwan Gaya, Korea (I). Journal of Conservation Science, 37(3): 232-244.

Kim, G, Kim, JG, Kang, K and Yoo, WS (2019) Image-Based Quantitative Analysis of Foxing Stains on Old Printed Paper Documents. Heritage, 2(3): 2665-2677.

Lee, MY and Wi, KC (2021) A Study on the Color of Natural Solvent for the Red Color Reproduction of Safflower. Journal of Conservation Science, 37(1): 13-24.

National Museum of Korea (2012) Portrait of Kang Io by Yi Jaegwan (1783-1837). Available at: https://en.wikipedia.org/wiki/Korean_painting\#/media/File:\%EC\%95\%BD\%EC\%82\%B0_ \%EA\%B0\%95\%EC\%9D\%B4\%EC\%98\%A4_\%EC\%B4\%88\%EC\%83\%81.jpg [accessed 9 July 2021]. 
National Museum of Korea (2021) Portrait of Kang Io. Available at: https://www.museum.go.kr/site/eng/relic/represent/view?relicId=1236 [accessed 9 July 2021].

Nix Color Sensor (2021) How do you Measure Color Accuracy? Available at: https://www.nixsensor.com/blog/measure-color-accuracy/ [accessed October 8, 2021].

Schuessler, Z (2021) Delta E 101. Available at: http://zschuessler.github.io/DeltaE/learn/ [accessed October 8, 2021].

Sharma, G (2003) Digital Color Imaging Handbook. Boca Raton: CRC Press.

Typepad (2021) REMOVING FOXING AND MILDEW. Available at: https://periodfinebindings.typepad.com/removing_foxing_and_milde/ [accessed 9 July 2021].

Yoo, WS and Kim, G (2017) An Application of Image Analysis in Conservation Science (I). Traditional Techniques and Modern Technology (The Proceeding of the Sixth Symposium of the Society for Conservation of Cultural Heritage in East Asia), 2017 Fudan University, Shanghai, China. Society for the Conservation of Cultural Heritage), 359-366.

Yoo, WS, Kang, K, Kim, JG and Jung, Y-H (2020) Development of Image Analysis Software for Archaeological Applications. In: NH Tan (ed.) Advancing Southeast Asian Archaeology 2019: Selected Papers from the Third SEAMEO SPAFA International Conference on Southeast Asian Archaeology. Bangkok: SEAMEO SPAFA, 402-411.

Yoo, WS, Kim, JG and Kim, G (2019) An Application of Image Analysis in Conservation Science (II). Proceeding of the Seventh Symposium of the Society for Conservation of Cultural Heritage in East Asia, Daejeon, Korea, 29-31 August 2019, 2019 Daejon, Korea. Society for the Conservation of Cultural Heritage, 20-24.

Yoo, WS (2020) Comparison of Outlines by Image Analysis for Derivation of Objective Validation Results: "Ito Hirobumi's Characters on the Foundation Stone" of the Main Building of Bank of Korea. Journal of Conservation Science, 36(6): 511-518.

Yoo, WS, Yoo, SS, Yoo, BH and Yoo, SJ (2021) Investigation on the Conservation Status of the 50-year-old "Yu Kil-Chun Archives" and an Effective and Practical Method of Preserving and Sharing Contents. Journal of Conservation Science, 37(2): 167-178.

Yoo, Y and Yoo, WS (2021) Digital Image Comparisons for Investigating Aging Effects and Artificial Modifications Using Image Analysis Software. Journal of Conservation Science, 37(1): 1-12. 